\title{
INVERSE COEFFICIENT PROBLEMS FOR A TRANSPORT EQUATION BY LOCAL CARLEMAN ESTIMATE
}

\author{
P. CANNARSA ${ }^{1}$, G. FLORIDIA ${ }^{2}$, F. GÖLGELEYEN ${ }^{3}$, M. YAMAMOTO ${ }^{4}$
}

\begin{abstract}
We consider the transport equation $\partial_{t} u(x, t)+(H(x) \cdot \nabla u(x, t))+p(x) u(x, t)=0$ in $\Omega \times(0, T)$ where $\Omega \subset \mathbb{R}^{n}$ is a bounded domain, and discuss two inverse problems which consist of determining a vector-valued function $H(x)$ or a real-valued function $p(x)$ by initial values and data on a subboundary of $\Omega$. Our results are conditional stability of Hölder type in a subdomain $D$ provided that the outward normal component of $H(x)$ is positive on $\partial D \cap \partial \Omega$. The proofs are based on a Carleman estimate where the weight function depends on $H$.
\end{abstract}

\section{INTRODUCTION AND MAIN RESULTS}

Let $\Omega \subset \mathbb{R}^{n}$ be a bounded domain with smooth boundary and $H=\left(h_{1}, \ldots, h_{n}\right) \in\left(C^{1}(\bar{\Omega})\right)^{n}$. Let $(x \cdot y)$ and $|x|$ denote the scalar product of $x, y \in \mathbb{R}^{n}$ and the norm of $x \in \mathbb{R}^{n}$, respectively. Let $u \in C^{1}(\bar{\Omega} \times[0, T])$ satisfy

$$
P u(x, t):=\partial_{t} u+(H(x) \cdot \nabla u)+p(x) u(x, t)=0 \quad \text { in } \Omega \times(0, T)
$$

with

$$
u(x, 0)=a(x), \quad x \in \Omega,
$$

where $a \in C^{1}(\bar{\Omega})$.

Fixing $p \in C(\bar{\Omega})$, by $u(H, a)=u(H, a)(x, t)$, we denote one solution to (1.1) and (1.2). We note that there exist many solutions and $u(H, a)$ is an arbitrarily chosen solution.

We discuss the following.

\section{Inverse coefficient problem.}

We fix $p$. Let $\Gamma \subset \partial \Omega$ be a subboundary and let $a_{1}, \ldots, a_{n}$ be chosen suitably. Then determine

1 Piermarco Cannarsa, Department of Mathematics, University of Rome "Tor Vergata", 00133 Rome, Italy, email: cannarsa@mat.uniroma2.it. $\quad{ }^{2}$ Giuseppe Floridia, Department of Mathematics and Applications "R. Caccioppoli", University of Naples Federico II, 80126 Naples, Italy, email: giuseppe.floridia@unina.it \& floridia.giuseppe@icloud.com. ${ }^{3}$ Fikret Gölgeleyen, Department of Mathematics, Bulent Ecevit University Zonguldak, 67100 Turkey, e-mail: golgeleyen@yahoo.com.

${ }^{4}$ Masahiro Yamamoto, Department of Mathematical Sciences, The University of Tokyo, Komaba, Meguro, Tokyo 153, Japan, Peoples' Friendship University of Russia (RUDN University), 6 Miklukho-Maklaya St, Moscow, 117198, Russian Federation, e-mail: myama@ms.u-tokyo.ac.jp. 
$H(x)=\left(h_{1}(x), \ldots, h_{n}(x)\right)$ near $\Gamma$ by boundary data $\left.u\left(H, a_{k}\right)\right|_{\Gamma \times(0, T)}, k=1,2, \ldots, n$.

Our problem is an inverse coefficient problem where spatially varying coefficients should be determined by lateral boundary data and initial data.

An equation of type (1.1) is a transport equation and often appears in mathematical physics. For example, let $H(x, t)$ be the velocity field of some fluid and let $u$ be the fluid density. Then conservation of mass yields

$$
\partial_{t} u+\operatorname{div}(u H)=0 \quad \text { in } \Omega \times(0, T),
$$

that is,

$$
\partial_{t} u+(H \cdot \nabla u)+(\operatorname{div} H) u=0 \quad \text { in } \Omega \times(0, T),
$$

which corresponds to (1.1) if $H$ is independent of $t$. We can interpret our inverse problem as the determination of a velocity field by boundary data on $\Gamma \times(0, T)$ and initial data.

In this paper, aiming at generous conditions for unknown $H$, we mainly discuss uniqueness and stability locally near $\Gamma$. More precisely, we are concerned with the existence of a subdomain $D \subset \Omega$ satisfying $\partial D \cap \partial \Omega \subset \Gamma$ where data $\left.u\left(H, a_{k}\right)\right|_{\Gamma \times(0, T)}$ with $k=1, \ldots, n$ stably determine $H$ in $D$.

In order to state our main results, we introduce some notations. Let $\nu=\nu(x)$ be the outward unit normal vector to $\partial \Omega$ at $x$. For arbitrarily fixed $x_{0} \in \Gamma$ and constants $\delta_{0}>$ $0, M>0$, we define the admissible set $\mathcal{H}$ by

$$
\begin{aligned}
\mathcal{H}= & \left\{H \in\left(C^{1}(\bar{\Omega})\right)^{n} ;\|H\|_{\left(C^{1}(\bar{\Omega})\right)^{n}} \leq M,\right. \\
& \left.\left(H\left(x_{0}\right) \cdot \nu\left(x_{0}\right)\right)>\delta_{0}, \quad|H(x)|>\delta_{0} \quad \text { for } x \in \Omega\right\} .
\end{aligned}
$$

Let ${ }^{T}$ denote the transpose of a generic vector and set $\nabla a(x)=\left(\partial_{1} a, \ldots, \partial_{n} a\right)^{T}$ for any realvalued function $a(x)$.

Henceforth, we always assume that $\partial_{t} u\left(H_{j}, a_{k}\right) \in C^{1}(\bar{\Omega} \times[0, T])$ for $j=1,2$ and $k=1, \ldots, n$.

We are ready to state our first main result.

\section{Theorem 1 (local stability for the inverse coefficient problem).}

We assume

$$
\left|\operatorname{det}\left(\nabla a_{1}(x), \ldots, \nabla a_{n}(x)\right)\right| \neq 0 \quad x \in \bar{\Omega}
$$

and

$$
\partial_{t} u\left(H_{j}, a_{k}\right) \in C^{1}(\bar{\Omega} \times[0, T]),\left\|\partial_{t} \nabla u\left(H_{j}, a_{k}\right)\right\|_{L^{2}\left(0, T ; L^{\infty}(\Omega)\right)} \leq M_{0}, j=1,2, k=1, \ldots, n
$$

for some constant $M_{0}>0$. Then there exist constants $C>0, \theta \in(0,1)$ and a subdomain $D \subset \Omega$ satisfying $x_{0} \in \partial D \cap \partial \Omega \subset \Gamma$ and such that

$$
\begin{aligned}
& \left\|H_{1}-H_{2}\right\|_{L^{2}(D)} \\
\leq & C\left(\sum_{k=1}^{n}\left\|\partial_{t} u\left(H_{1}, a_{k}\right)-\partial_{t} u\left(H_{2}, a_{k}\right)\right\|_{L^{2}(\Gamma \times(0, T))}^{\theta}+\sum_{k=1}^{n}\left\|\partial_{t} u\left(H_{1}, a_{k}\right)-\partial_{t} u\left(H_{2}, a_{k}\right)\right\|_{L^{2}(\Gamma \times(0, T))}\right)
\end{aligned}
$$


for $H_{1}, H_{2} \in \mathcal{H}$. Here the constants $C$ and $\theta$ depend on $T, \Gamma, \mathcal{H}$ and $M_{0}$, while $D$ depends on $T, \Gamma$ and $\mathcal{H}$, but it is independent of choices of $H_{1}, H_{2}$.

Since $H(x)=\left(h_{1}(x), \ldots, h_{n}(x)\right)$ possesses $n$ unknown components, we need to take $n$ different measurements of the initial values satisfying (1.4) and observe boundary data. We do not pose any assumptions on the size of $\Gamma$ and $T>0$, and the finiteness of the propagation speed of (1.1) forces the domain $D$ in the stability result to be in general smaller if $T>0$ is small, which can be seen from the proof.

Theorem 1 provides a conditional stability estimate of Hölder type for the inverse coefficient problem and asserts that we can estimate $H_{1}-H_{2}$ provided that $H_{1}, H_{2} \in \mathcal{H}$ and condition(1.5) is satisfied.

Theorem 1 directly yields the following.

\section{Corollary (local uniqueness for the inverse coefficient problem).}

We assume (1.4). Then there exists a subdomain $D \subset \Omega$ satisfying $x_{0} \in \partial D \cap \partial \Omega \subset \Gamma$ such that, if

$$
\left.u\left(H_{1}, a_{k}\right)\right|_{\Gamma \times(0, T)}=\left.u\left(H_{2}, a_{k}\right)\right|_{\Gamma \times(0, T)}, \quad k=1,2, \ldots, n
$$

with $H_{1}, H_{2} \in \mathcal{H}$, then $H_{1}(x)=H_{2}(x)$ for all $x \in D$.

We can prove similar uniqueness and stability results for the inverse problem of determining $p(x)$ in (1.1). We fix $H \in \mathcal{H}$ arbitrarily and denote by $u(p, a)$ one solution to (1.1) and (1.2). Theorem 2 (local stability for the inverse coefficient problem). For arbitrarily fixed constant $M>0$, we set

$$
\mathcal{P}:=\left\{p \in L^{\infty}(\Omega) ;\|p\|_{L^{\infty}(\Omega)} \leq M\right\} .
$$

We assume

$$
|a(x)| \neq 0, \quad x \in \bar{\Omega},
$$

$\partial_{t} u\left(p_{j}, a\right) \in C^{1}(\bar{\Omega} \times[0, T]), j=1,2$, and

$$
\left\|\partial_{t} u\left(p_{j}, a\right)\right\|_{L^{2}\left(0, T ; L^{\infty}(D)\right)} \leq M_{0}, \quad j=1,2,
$$

for some constant $M_{0}>0$. Then there exist a subdomain $D \subset \Omega$ satisfying $x_{0} \in \partial D \cap \partial \Omega \subset \Gamma$ and constants $C>0$ and $\theta \in(0,1)$, depending on $T, \Gamma, \mathcal{H}$ and $M_{0}$, such that

$$
\begin{aligned}
& \left\|p_{1}-p_{2}\right\|_{L^{2}(D)} \\
\leq & C\left(\left\|\partial_{t} u\left(p_{1}, a\right)-\partial_{t} u\left(p_{2}, a\right)\right\|_{L^{2}(\Gamma \times(0, T))}^{\theta}+\left\|\partial_{t} u\left(p_{1}, a\right)-\partial_{t} u\left(p_{2}, a\right)\right\|_{L^{2}(\Gamma \times(0, T))}\right)
\end{aligned}
$$

for $p_{1}, p_{2} \in \mathcal{P}$ satisfying (1.7). Here $D$ depends on $T, \Gamma$ and $\mathcal{H}$, but it is independent of the choice of $p_{1}, p_{2}$.

In Theorems 1 and 2, we do not know boundary values on $\partial D \backslash \Gamma$, and Lemma 2 in Section 2 proves that $(H \cdot \nu) \leq 0$ on $\partial D \backslash \Gamma$, where $\nu$ is the outward unit normal vector to $D$. Therefore, in terms of data on the inflow and outflow subboundaries, we note that we cannot uniquely determine the solutions $u\left(H_{j}, a_{k}\right), u\left(p_{j}, a\right)$ themselves on the whole domain 
$D \times(0, T)$ although the coefficients are uniquely determined in $D$. In fact, for the uniqueness of solution $u$ in $D \times(0, T)$, data of $u$ on $\{x \in \partial D ; H(x) \cdot \nu(x) \leq 0\}$ are indispensable. We give an illustrating simple example:

Example.

$$
\left\{\begin{array}{l}
\partial_{t} u(x, t)-\partial_{x} u(x, t)=0, \quad 0<x, t<1 \\
u(x, 0)=0, \quad 0<x<1 \\
u(0, t)=0, \quad 0<t<1
\end{array}\right.
$$

We can regard $u$ as the difference of two solutions of (1.1) with $p=0$ satisfying (1.2) with same $a$. Let $g \not \equiv 0$ be a function of class $C^{1}(\mathbb{R})$ satisfying $g(\eta)=0$ for $\eta \leq 1$. Then we can easily see that $u(x, t)=g(x+t)$ is a solution to the above initial-boundary value problem in $(0,1)^{2}$. This example indicates that no uniqueness result can be expected for the solution $u$ in the whole domain. Here $H(0)=-1$ and $\nu(0)=-1$ at $x=0$ where boundary data are given, and we note that $(H(0) \cdot \nu(0))=1>0$, but we are given no boundary data on the subboundary where $(H(1) \cdot \nu(1))<0$.

In particular, if we know data of $u$ on $(\partial D \backslash \Gamma) \times(0, T)$, then we can prove that in the inverse problem, both coefficients and solution are uniquely determined.

Since (1.1) is a first order equation, for the above inverse problem one could apply the method of characteristics as was done, for example, by Belinskij [2] and Romanov [21, Chapter 5]. However, in this paper we follow a different approach because

(1) the method of characteristics does not directly provide stability estimates in $L^{2}$-spaces (like Theorems 1 and 2) for inverse problems;

(2) for inverse coefficient problems, a more comprehensive method is available, which is applicable not only to hyperbolic, parabolic, Schrödinger, and other equations in mathematical physics, but also to the transport equation.

Such a comprehensive methodology for inverse coefficient problems was created by Bukhgeim and Klibanov [4]. Since [4], there have been many publications for second-order equations. We can refer for example to Beilina and Klibanov [1], Bellassoued and Yamamoto [3], Imanuvilov and Yamamoto [9] - [12, Klibanov [14, [15], Klibanov and Timonov [18, Klibanov and Yamamoto [19], Yamamoto [22]. Here we do not intend to give any substantial list of references and the reader can consult the references contained in these works. The methodology by Bukhgeim and Klibanov [4] is based on Carleman estimates which are $L^{2}$ weighted estimates for solutions to partial differential equations. The choice of the weight functon is crucial for each Carleman estimate. As for Carleman estimates themselves, see Hörmander [8], Isakov [13] for instance.

Especially for transport equations, the approach based on Carleman estimates was adopted, for instance, in Cannarsa, Floridia and Yamamoto [5], Gaitan and Ouzzane [6], and Gölgeleyen and Yamamoto [7]. The works [6] and [7] discuss global stability in $x$ provided that boundary values are given on the whole lateral boundary $\partial \Omega \times(0, T)$. Our approach is similar to Gölgeleyen and Yamamoto [7] but, in this paper, by concentrating on local stability we can allow the unknown principal part to satisfy the generous condition $\left(H\left(x_{0}\right) \cdot \nu\left(x_{0}\right)\right)>0$. Reference [5] considers a transport equation $\partial_{t} u+(H(t) \cdot \nabla u)=0$ with time-dependent $H(t)$ 
and establishes a Carleman estimate to prove an observability-type inequality for the $L^{2}$ norm of the initial value in terms of boundary data. Moreover, Klibanov and Pamyatnykh [16], 17] and Machida and Yamamoto [20] discuss Carleman estimates and inverse problems for a transport equation including an integral term with respect to a velocity field.

In our method, the choice of the weight function in our Carleman estimate is essential for the application to inverse problems. The weight function of our Carleman estimate (Proposition 1 in Section 2) is linear in $t$ and similar to [7], but different from [6], [16], [17] where the weight is quadratic in $t$. Thanks to our choice of the weight function, we need not extend the solution $u$ to $(-T, 0)$. Moreover, as is already explained, in our Carleman estimate, the sufficient condition $\left(H\left(x_{0}\right) \cdot \nu\left(x_{0}\right)\right)>0$ in (1.3) seems more related to a physical interpretation than the one in [6]. Furthermore, for second order hyperbolic equations one can take even or odd extensions of the solution $u(x, t)$ to establish Carleman estimates in $\Omega \times(-T, T)$. We notice that a Carleman estimate in $(-T, T)$ is usually easier to be established than on $\Omega \times(0, T)$. For the transport equation such extensions cause a difficulty because the signs of the principal terms are not the same in $(0, T)$ and $(-T, 0)$. We note that by starting with $(1.1)$ in $t \in(-T, T)$ with (1.2) and not extending in $t$, the inverse problem is easier but this is not an inverse problem for an initial-boundary value problem, and so it is unnatural to assume that the choice of $a, a_{k}$ is given a priori.

To sum up, compared with the existing related papers [2], [6], [7], [21] on inverse problems for the transport equation (1.1), we list our achievements:

- inverse problems for initial-boundary value problems;

- local stability in $x$ : this means that we do not assume boundary data on the whole lateral boundary $\partial \Omega \times(0, T)$ and we are concerned with estimation in a small subdomain;

- flexible choice of the weight function for the Carleman estimate which essentially depends on $H(x)$;

- estimates in $L^{2}$-norms unlike [2] and [21].

This paper is composed of five sections. In Section 2, we establish a key Carleman estimate (Proposition 1) and a lemma characterizing the subdomain $D$. Sections 3 and 4 are devoted to the proofs of Theorems 1 and 2, respectively, and Section 5 is devoted to remarks about other possible formulations of inverse problems.

\section{Key Carleman estimate And a Key lemma}

Let $D \subset \Omega$ be a bounded domain and let $Q=D \times(0, T)$, and $\nu=\nu(x)$ be the outward unit normal vector to $\partial D$ at $x$. Without loss of generality, we assume

$$
0 \in \bar{D} \text {. }
$$

We set

$$
\begin{aligned}
\psi_{H}(x) & =\sum_{j=1}^{n} x_{j} h_{j}(x), \quad x \in D, \\
\mu_{H}: & =\min _{x \in \bar{D}}\left(H(x) \cdot \nabla \psi_{H}(x)\right),
\end{aligned}
$$


and

$$
\begin{aligned}
& \Gamma_{+}=\{x \in \partial D ;(H(x) \cdot \nu(x)) \geq 0\} \\
& \Gamma_{-}=\{x \in \partial D ;(H(x) \cdot \nu(x)) \leq 0\}
\end{aligned}
$$

We can prove

\section{Lemma 1.}

Let $H \in \mathcal{H}$ be arbitrary. If diam $D<\frac{\delta_{0}^{2}}{2 M^{2}}$, then

$$
\mu:=\inf _{H \in \mathcal{H}} \mu_{H} \geq \frac{\delta_{0}^{2}}{2} .
$$

\section{Proof.}

Let $H=\left(h_{1}, \ldots, h_{n}\right) \in \mathcal{H}$ be arbitrary. By the Cauchy-Schwarz inequality, we have

$$
\begin{aligned}
& \left(H(x) \cdot \nabla \psi_{H}(x)\right)=\sum_{k=1}^{n} h_{k}(x)^{2}+\sum_{k=1}^{n} h_{k}(x) \sum_{j=1}^{n} x_{j} \partial_{k} h_{j}(x) \\
\geq \quad & \min _{x \in \bar{D}}|H(x)|^{2}-\left(\sum_{k=1}^{n} h_{k}(x)^{2}\right)^{\frac{1}{2}}\left(\sum_{k=1}^{n}\left|\sum_{j=1}^{n} x_{j} \partial_{k} h_{j}(x)\right|^{2}\right)^{\frac{1}{2}} \\
\geq & \delta_{0}^{2}-\|H\|_{\left(L^{\infty}(D)\right)^{n}}\left(\sum_{k=1}^{n}\left(\sum_{j=1}^{n}\left|x_{j}\right|^{2}\right)\left(\sum_{j=1}^{n}\left|\partial_{k} h_{j}(x)\right|^{2}\right)\right)^{\frac{1}{2}} \\
\geq & \delta_{0}^{2}-\max _{x \in \bar{D}}|x|\|H\|_{\left(L^{\infty}(D)\right)^{n}}\|\nabla H\|_{\left(L^{\infty}(D)\right)^{n \times n} \geq \delta_{0}^{2}-(\operatorname{diam} D) M^{2} .}
\end{aligned}
$$

Therefore $\inf _{H \in \mathcal{H}} \mu_{H} \geq \frac{\delta_{0}^{2}}{2}$ if diam $D<\frac{\delta_{0}^{2}}{2 M^{2}}$. Thus the proof of Lemma 1 is complete.

We further set

$$
\varphi_{H}(x, t)=-\beta t+\psi_{H}(x), \quad(x, t) \in Q, \quad B_{H}(x)=\left(H(x) \cdot \nabla \psi_{H}(x)\right)-\beta,
$$

where $\beta>0$ satisfies $0<\beta<\mu$. Then (2.2) implies

$$
B_{H}(x) \geq \mu-\beta>0, \quad x \in \bar{D} .
$$

We can state the key Carleman estimate.

\section{Proposition 1 (Carleman estimate).}

Let $\partial D$ be piecewise smooth. There exist constants $s_{0}>0$ and $C>0$ such that

$$
\begin{aligned}
& s \int_{D}|u(x, 0)|^{2} e^{2 s \varphi_{H}(x, 0)} d x+s^{2} \int_{Q}|u|^{2} e^{2 s \varphi_{H}(x, t)} d x d t+C e^{-C s} \int_{\Gamma_{-} \times(0, T)}|(H \cdot \nu)||u|^{2} d S d t \\
\leq & C \int_{Q}|P u|^{2} e^{2 s \varphi_{H}(x, t)} d x d t+C e^{C s} \int_{\Gamma_{+} \times(0, T)}|u|^{2} d S d t+C s \int_{D}|u(x, T)|^{2} e^{2 s \varphi_{H}(x, T)} d x
\end{aligned}
$$

for all $s>s_{0}$, all $H \in \mathcal{H}, p \in \mathcal{P}$ and $u \in H^{1}(Q)$. 
We note that $C>0$ and $s_{0}>0$ are independent of choices of $H \in \mathcal{H}$ and $p \in \mathcal{P}$.

\section{Proof of Proposition 1.}

It suffices to assume that $p \equiv 0$. Indeed let the proposition be proved for $p \equiv 0$. Then, applying (2.4) to $\partial_{t} u+(H \cdot \nabla u)=P u-p u$, we have

$$
\begin{aligned}
& s \int_{D}|u(x, 0)|^{2} e^{2 s \varphi_{H}(x, 0)} d x+s^{2} \int_{Q}|u|^{2} e^{2 s \varphi_{H}(x, t)} d x d t+C e^{-C s} \int_{\Gamma_{-} \times(0, T)}|(H \cdot \nu)||u|^{2} d S d t \\
\leq & C \int_{Q}|P u|^{2} e^{2 s \varphi_{H}(x, t)} d x d t+C \int_{Q}|p u|^{2} e^{2 s \varphi_{H}(x, t)} d x d t+C e^{C s} \int_{\Gamma_{+} \times(0, T)}|u|^{2} d S d t \\
+ & C s \int_{D}|u(x, T)|^{2} e^{2 s \varphi_{H}(x, T)} d x .
\end{aligned}
$$

Since $\|p\|_{L^{\infty}(\Omega)} \leq M$ by $p \in \mathcal{P}$, we can absorb the second term on the right-hand side into the left-hand side by choosing $s>0$ large, so that (2.4) is seen to hold for general $p \in \mathcal{P}$. Let

$$
w=e^{s \varphi_{H}} u, \quad L w=e^{s \varphi_{H}} P\left(e^{-s \varphi_{H}} w\right) .
$$

Then

$$
L w=\partial_{t} w+(H(x) \cdot \nabla w)-s B_{H} w \quad \text { in } Q .
$$

Therefore, in terms of (2.3), we have

$$
\begin{aligned}
& \int_{0}^{T} \int_{D}|P u|^{2} e^{2 s \varphi_{H}} d x d t=\int_{0}^{T} \int_{D}|L w|^{2} d x d t \\
\geq & -2 s \int_{Q} B_{H} w\left(\partial_{t} w+(H(x) \cdot \nabla w)\right) d x d t+s^{2} \int_{Q}(\mu-\beta)^{2}|w|^{2} d x d t \\
= & -s \int_{Q} B_{H} \partial_{t}\left(|w|^{2}\right) d x d t-s \int_{Q} B_{H} \sum_{j=1}^{n} h_{j} \partial_{j}\left(|w|^{2}\right) d x d t \\
+ & s^{2}(\mu-\beta)^{2} \int_{Q}|w|^{2} d x d t \\
= & s \int_{D} B_{H}(x)\left[|w(x, t)|^{2}\right]_{t=T}^{t=0} d x+s \int_{Q} \operatorname{div}\left(B_{H} H\right)|w|^{2} d x d t \\
- & s \int_{0}^{T} \int_{\partial D} B_{H}(x)(H \cdot \nu)|w|^{2} d S d t+s^{2}(\mu-\beta)^{2} \int_{Q}|w|^{2} d x d t \\
\geq & s \int_{D}(\mu-\beta)|w(x, 0)|^{2} d x-C s \int_{D}|w(x, T)|^{2} d x+s \int_{Q} \operatorname{div}\left(B_{H} H\right)|w|^{2} d x d t \\
- & s \int_{\Gamma_{+} \times(0, T)} B_{H}(x)(H \cdot \nu)|w|^{2} d S d t-s \int_{\Gamma_{-} \times(0, T)} B_{H}(x)(H \cdot \nu)|w|^{2} d S d t \\
+ & s^{2}(\mu-\beta)^{2} \int_{Q}|w|^{2} d x d t .
\end{aligned}
$$


Hence, using $\sup _{H \in \mathcal{H}}\left\|\varphi_{H}\right\|_{C(\bar{D})}<\infty$ and taking some constant $C(M)>0$, we obtain

$$
\begin{aligned}
& \int_{Q}|P u|^{2} e^{2 s \varphi_{H}} d x d t+C s \int_{\Gamma_{+} \times(0, T)}|u|^{2} e^{2 s \varphi_{H}} d S d t+C s \int_{D}|u(x, T)|^{2} e^{2 s \varphi_{H}(x, T)} d x \\
\geq & s \int_{D}(\mu-\beta)|u(x, 0)|^{2} e^{2 s \varphi_{H}(x, 0)} d x+s^{2}\left((\mu-\beta)^{2}-\frac{C(M)}{s}\right) \int_{Q}|w|^{2} d x d t \\
+ & C s \int_{\Gamma_{-} \times(0, T)}\left|B_{H}(x)(H \cdot \nu)\right||u|^{2} e^{2 s \varphi_{H}} d S d t .
\end{aligned}
$$

Here $C(M)>0$ is dependent on $M$, but independent of choices of $p \in \mathcal{P}$ and $H \in \mathcal{H}$, and we can choose $s_{0}>0$ and $C>0$ independently of the choices of $p$ and $H$, such that (2.4) holds. Thus the proof of Proposition 1 is completed.

We conclude this section with a lemma characterizing the subdomain $D$ which can be arbitralily small as we wish.

Lemma 2. There exists a constant $r>0$ such that for $0<\varepsilon<r$, we can choose a subdomain $D=D(\varepsilon) \subset \Omega$ such that $\partial D$ is piecewise smooth, diam $D<\varepsilon$ and

$$
\begin{gathered}
\partial D \cap \partial \Omega \supset \Gamma \cap\left\{x \in \mathbb{R}^{n} ;|x-\widetilde{x}|<\rho_{0}\right\} \\
\text { with some } \widetilde{x} \in \mathbb{R}^{n} \text { and } \rho_{0}>0, x_{0} \in \partial D \cap \partial \Omega \text {, and } \\
(H(x) \cdot \nu(x)) \leq 0 \quad \text { for all } x \in \partial D \backslash \Gamma \text { and all } H \in \mathcal{H} .
\end{gathered}
$$

\section{Proof.}

First Step. It suffice to prove that there exist $r>0$ and $D$ satisfying $(2.5)$ and diam $D<r$. Then by the argument below, we see that for small $\varepsilon>0$ satisfying $0<\varepsilon<r$, there exists a domain $D(\varepsilon)$ satisfying $(2.5)$.

We set $x=\left(x^{\prime}, x_{n}\right) \in \mathbb{R}^{n-1} \times \mathbb{R}, \nabla^{\prime} \ell\left(x^{\prime}\right)=\left(\partial_{1} \ell, \ldots, \partial_{n-1} \ell\right)^{T}, B_{\rho}=\left\{x^{\prime} \in \mathbb{R}^{n-1} ;\left|x^{\prime}\right|<\rho\right\}$ with $\rho>0$. For a domain $E \subset \mathbb{R}^{n}$ and subboundary $L \subset \partial E$, by $\nu_{L}(x)$ we denote the outward unit normal vector to $E$ at $x \in L$.

Since we assume that $x_{0}=0$, from (1.3) it follows that

$$
\left(H(0) \cdot \nu_{\Gamma}(0)\right)>\delta_{0} \quad \text { for all } H \in \mathcal{H} .
$$

Since $\partial \Omega$ is smooth, there exist a constant $\rho_{0}>0$ and a smooth function $\ell$ defined in $B_{\rho_{0}}$ such that $\ell(0)=0$ and

$$
\gamma_{0}=\left\{\left(x^{\prime}, x_{n}\right) ; x^{\prime} \in B_{\rho_{0}}, \quad x_{n}=\ell\left(x^{\prime}\right)\right\} \subset \partial \Omega .
$$

Without loss of generality, we further assume that $\Omega$ is located below $x_{n}=\ell\left(x^{\prime}\right)$ locally near 0 , that is, if $\left|x^{\prime}\right|$ is small and $x_{n}-\ell\left(x^{\prime}\right)$ is positive and small, then $\left(x^{\prime}, x_{n}\right) \notin \bar{\Omega}$. Then we can represent

$$
\nu_{\gamma}\left(x^{\prime}, x_{n}\right)=\frac{\left(-\nabla^{\prime} \ell\left(x^{\prime}\right), 1\right)^{T}}{\sqrt{1+\left|\nabla^{\prime} \ell\left(x^{\prime}\right)\right|^{2}}}
$$


Then from the interval $\left(0, \min \left\{\rho_{0}, 1\right\}\right)$, we can choose a constant $\rho_{1}>0$ sufficiently small such that

$$
\begin{gathered}
\qquad\left(H(x) \cdot \nu_{\Gamma}(x)\right)>\frac{\delta_{0}}{2} \\
\text { for all } x \in \gamma_{1}:=\left\{\left(x^{\prime}, x_{n}\right) \in \mathbb{R}^{n} ; x_{n}=\ell\left(x^{\prime}\right),\left|x^{\prime}\right|<\rho_{1}\right\} \text { and all } H \in \mathcal{H} \text {. }
\end{gathered}
$$

We note that $0 \in \gamma_{1}$ and $\gamma_{1} \subset \gamma_{0}$, and $\rho_{1}$ is independent of choices $H \in \mathcal{H}$.

Second Step: Proof of (2.6). As is easily verified, we have

$$
\sup _{x \in \gamma_{1}}|x| \leq \rho_{1}\left(1+\|\ell\|_{C^{1}(\partial \Omega)}^{2}\right)^{\frac{1}{2}}=: C(M) \rho_{1} .
$$

Henceforth $C=C(M)>0$ denotes generic constants which are independent of choices of $H \in \mathcal{H}$ and $x \in \mathbb{R}^{n}$ near 0 . Hence

$$
\begin{aligned}
& \left(H(x) \cdot \nu_{\Gamma}(x)\right)=\left(H(0) \cdot \nu_{\Gamma}(0)\right)+\left((H(x)-H(0)) \cdot \nu_{\Gamma}(x)\right)+\left(H(0) \cdot\left(\nu_{\Gamma}(x)-\nu_{\Gamma}(0)\right)\right. \\
\geq & \left(H(0) \cdot \nu_{\Gamma}(0)\right)-2 M|x| \sup _{x \in \gamma_{1}}\left|\nu(x)_{\Gamma}\right|-M \sup _{x \in \gamma_{1}}\left|\nu_{\Gamma}(x)-\nu_{\Gamma}(0)\right| \\
> & \delta_{0}-C(M) \rho_{1} .
\end{aligned}
$$

Therefore if $\rho_{1}<\frac{\delta_{0}}{2 C(M)}$, then $(2.6)$ is verified.

Next we choose $\tilde{\ell} \in C^{2}\left(\overline{B_{\rho_{1}}}\right)$ such that $\tilde{\ell}\left(x^{\prime}\right)>0$ for $\left|x^{\prime}\right|<\rho_{1}$ and $\tilde{\ell}\left(x^{\prime}\right)=0$ for $\left|x^{\prime}\right|=\rho_{1}$ and

$$
\|\widetilde{\ell}\|_{C^{2}\left(\overline{B_{\rho_{1}}}\right)} \leq \frac{\delta_{0}}{M}, \quad \partial_{i} \partial_{j} \widetilde{\ell}\left(x^{\prime}\right)=0, \quad 1 \leq i, j \leq n-1,\left|x^{\prime}\right|=\rho_{1} .
$$

We set

$$
D=\left\{\left(x^{\prime}, x_{n}\right) \in \mathbb{R}^{n} ; \ell\left(x^{\prime}\right)-\widetilde{\ell}\left(x^{\prime}\right)<x_{n}<\ell\left(x^{\prime}\right), \quad\left|x^{\prime}\right|<\rho_{1}\right\}
$$

and

$$
\gamma_{2}=\left\{\left(x^{\prime}, x_{n}\right) \in \mathbb{R}^{n} ; x_{n}=\ell\left(x^{\prime}\right)-\tilde{\ell}\left(x^{\prime}\right), \quad\left|x^{\prime}\right|<\rho_{1}\right\} .
$$

Then we see that $D \backslash \overline{\gamma_{1}} \subset \Omega$ and $\partial D=\gamma_{1} \cup \gamma_{2}$.

Now we prove

$$
\left(H(x) \cdot \nu_{\gamma_{2}}(x)\right) \leq-\frac{\delta_{0}}{4} \quad \text { for all } x \in \gamma_{2} \text { and all } H \in \mathcal{H} .
$$

We note that $\left(H(x) \cdot \nu_{\gamma_{k}}(x)\right), k=1,2$ may be discontinous on $\overline{\gamma_{1} \cap \gamma_{2}}$.

Third Step: Proof of (2.7). The outward normal vector $\nu_{\gamma_{2}}(x)$ to $D$ at $x \in \gamma_{2}$ is given by

$$
\nu_{\gamma_{2}}(x)=\frac{\left(\nabla^{\prime}(\ell-\tilde{\ell})\left(x^{\prime}\right),-1\right)^{T}}{\sqrt{1+\left|\nabla^{\prime}(\ell-\widetilde{\ell})\left(x^{\prime}\right)\right|^{2}}}
$$

if $\left|x^{\prime}\right|<\rho_{1}$. Then the definition of $\gamma_{1}$ and $\gamma_{2}$ yields

$$
\nu_{\gamma_{2}}\left(x^{\prime}, x_{n}\right)=-\nu_{\gamma_{1}}\left(x^{\prime}, x_{n}\right) \text { for }\left|x^{\prime}\right|=\rho_{1},
$$

and $D$ is the connected part between the two subboundaries $\gamma_{1}, \gamma_{2}$. For an arbitrarily fixed $y=\left(y^{\prime},(\ell-\widetilde{\ell})\left(y^{\prime}\right)\right) \in \gamma_{2}$ with $\left|y^{\prime}\right|=\rho_{1}$, we see from (2.6) and (2.8) that

$$
\left(H(y) \cdot \nu_{\gamma_{2}}(y)\right) \leq-\frac{\delta_{0}}{2} \quad \text { for all } H \in \mathcal{H}
$$


For each $x, y \in \gamma_{2}$, we have

$$
\begin{aligned}
& \quad\left|\nu_{\gamma_{2}}(x)-\nu_{\gamma_{2}}(y)\right|=\mid\left(\frac{1}{\sqrt{1+\left|\nabla^{\prime}(\ell-\tilde{\ell})\left(x^{\prime}\right)\right|^{2}}}-\frac{1}{\sqrt{1+\left|\nabla^{\prime}(\ell-\widetilde{\ell})\left(y^{\prime}\right)\right|^{2}}}\right)\left(\nabla^{\prime}(\ell-\tilde{\ell})\left(x^{\prime}\right),-1\right)^{T} \\
& +\frac{1}{\sqrt{1+\left|\nabla^{\prime}(\ell-\tilde{\ell})\left(y^{\prime}\right)\right|^{2}}}\left(\nabla^{\prime}(\ell-\tilde{\ell})\left(x^{\prime}\right)-\nabla^{\prime}(\ell-\tilde{\ell})\left(y^{\prime}\right), 0\right)^{T} \mid \\
& \leq C \frac{\left|\sqrt{1+\left|\nabla^{\prime}(\ell-\widetilde{\ell})\left(x^{\prime}\right)\right|^{2}}-\sqrt{1+\left|\nabla^{\prime}(\ell-\widetilde{\ell})\left(y^{\prime}\right)\right|^{2}}\right|}{\sqrt{1+\left|\nabla^{\prime}(\ell-\widetilde{\ell})\left(x^{\prime}\right)\right|^{2}} \sqrt{1+\left|\nabla^{\prime}(\ell-\widetilde{\ell})\left(y^{\prime}\right)\right|^{2}}} \\
& +C\left|\nabla^{\prime}(\ell-\tilde{\ell})\left(x^{\prime}\right)-\nabla^{\prime}(\ell-\tilde{\ell})\left(y^{\prime}\right)\right| \\
& \leq\left. C|| \nabla^{\prime}(\ell-\tilde{\ell})\left(x^{\prime}\right)\right|^{2}-\left|\nabla^{\prime}(\ell-\tilde{\ell})\left(y^{\prime}\right)\right|^{2}|+C| \nabla^{\prime}(\ell-\tilde{\ell})\left(x^{\prime}\right)-\nabla^{\prime}(\ell-\tilde{\ell})\left(y^{\prime}\right) \mid \\
& \quad<C \rho_{1} .
\end{aligned}
$$

At the last inequality, by the mean value theorem, we argued

$$
\begin{aligned}
& \left|\partial_{i}(\ell-\tilde{\ell})\left(x^{\prime}\right)-\partial_{i}(\ell-\tilde{\ell})\left(y^{\prime}\right)\right| \leq\left\|\nabla^{\prime} \partial_{i}(\ell-\tilde{\ell})\right\|_{C\left(\overline{B_{\rho_{1}}}\right)}\left|x^{\prime}-y^{\prime}\right| \\
\leq & \|\ell-\widetilde{\ell}\|_{C^{2}\left(\overline{B_{\rho_{1}}}\right)}\left(\left|x^{\prime}\right|+\left|y^{\prime}\right|\right) \leq 2 C \rho_{1}, \quad 1 \leq i \leq n-1
\end{aligned}
$$

for $x^{\prime}, y^{\prime} \in B_{\rho_{1}}$. Therefore (2.9) and (2.10) imply

$$
\begin{aligned}
& \left(H(x) \cdot \nu_{\gamma_{2}}(x)\right)=\left(H(y) \cdot \nu_{\gamma_{2}}(y)\right)+\left(H(y) \cdot\left(\nu_{\gamma_{2}}(x)-\nu_{\gamma_{2}}(y)\right)+\left((H(x)-H(y)) \cdot \nu_{\gamma_{2}}(x)\right)\right. \\
< & -\frac{\delta_{0}}{2}+\mid\left(H(y) \cdot\left(\nu_{\gamma_{2}}(x)-\nu_{\gamma_{2}}(y)\right)|+|\left((H(x)-H(y)) \cdot \nu_{\gamma_{2}}(x)\right) \mid\right. \\
\leq & -\frac{\delta_{0}}{2}+C M \rho_{1}+C M \rho_{1} .
\end{aligned}
$$

We further choose $\rho_{1}>0$ smaller such that $2 C M \rho_{1}<\frac{\delta_{0}}{4}$. Then $(2.7)$ is verified.

We can readily see that diam $D \leq \sqrt{2} \rho_{1}$, and setting $r \leq \sqrt{2} \rho_{1}$, we complete the proof of Lemma 2.

\section{Proof of Theorem 1.}

The proof is based on a key idea which is similar to Gölgeleyen and Yamamoto [7], but for our stability local in $x$ we need non-trivial modifications. The proof is divided into two steps.

First Step: Cut-off and separation of the range set of the weight function. Since $u\left(H_{j}, a_{k}\right)$ does not vanish at $t=T$, we cannot directly apply Proposition 1 . Therefore we need to introduce a cut-off function $\chi(t)$ vanishing near $t=T$ to consider $\chi\left(u\left(H_{1}, a_{k}\right)-\right.$ $\left.u\left(H_{2}, a_{k}\right)\right)$. However the transport equations satisfied by these functions which are cut off by $\chi$, contain some extra terms with $\frac{d \chi}{d t}$ and for keeping these terms as minor terms, we have to verify some separation of the range set of the weight function $\varphi_{H_{1}}(x, t)$. 
In (1.3), for simplicity, we can assume that $x_{0}=0$. Let $H, H_{1}, H_{2} \in \mathcal{H}$ be arbitrary. We choose $\beta>0$ satisfying

We choose $\varepsilon>0$ satisfying

$$
0<\beta<\frac{\delta_{0}^{2}}{2}
$$

$$
0<\varepsilon<\min \left\{\frac{\delta_{0}^{2}}{2 M^{2}}, 1, \frac{\beta T}{4 M}, r\right\},
$$

where $r>0$ is the constant whose existence is guaranteed by Lemma 2. By Lemma 2, we can construct a small domain $D$ such that $\partial D \cap \partial \Omega$ is a non-empty subset of the interior of $\Gamma, 0 \in \partial D \cap \partial \Omega$, diam $D<\varepsilon$ and $(H(x) \cdot \nu(x)) \leq 0$ for all $x \in \partial D \backslash \Gamma$ and all $H \in \mathcal{H}$. Then, by (3.1) Lemma 1 implies that

$$
\mu:=\inf _{H \in \mathcal{H}} \min _{x \in \bar{D}}\left(H(x) \cdot \nabla \psi_{H}(x)\right) \geq \frac{\delta_{0}^{2}}{2} .
$$

Therefore Carleman estimate (2.4) holds in $Q=D \times(0, T)$ uniformly with respect to $H \in \mathcal{H}$.

Next we estimate $\max _{y \in \bar{D}} \psi_{H}(y)-\min _{y \in \bar{D}} \psi_{H}(y)$. We can choose $\xi=\xi(H), \eta=\eta(H) \in D$ such that

$$
\max _{y \in \bar{D}} \psi_{H}(y)-\min _{y \in \bar{D}} \psi_{H}(y)=\psi_{H}(\xi)-\psi_{H}(\eta) .
$$

Hence, since $\|H\|_{\left(C^{1}(\bar{D})\right)^{n}} \leq M$ by $H \in \mathcal{H}$ and $M \operatorname{diam} D<\varepsilon M \leq M$ by (3.1), the mean value theorem yields

$$
\begin{aligned}
& \max _{y \in \bar{D}} \psi_{H}(y)-\min _{y \in \bar{D}} \psi_{H}(y) \leq\left\|\nabla \psi_{H}\right\|_{(C(\bar{D}))^{n}}|\xi-\eta| \\
\leq & \max _{1 \leq k \leq n}\left\|h_{k}+\sum_{j=1}^{n}\left(\partial_{k} h_{j}\right) x_{j}\right\|_{C(\bar{D})} \max _{y, y^{\prime} \in \bar{D}}\left|y-y^{\prime}\right| \leq(M+M \operatorname{diam} D) \operatorname{diam} D \leq 2 M \operatorname{diam} D .
\end{aligned}
$$

Again condition (3.1) implies

$$
\max _{y \in \bar{D}} \psi_{H}(y)-\min _{y \in \bar{D}} \psi_{H}(y)<2 M \varepsilon \leq 2 M \frac{\beta T}{4 M}=\frac{1}{2} \beta T,
$$

for all $H \in \mathcal{H}$. By (3.2), for all $H \in \mathcal{H}$, we obtain

$$
\begin{aligned}
& \min _{y \in \bar{D}} \varphi_{H}(y, 0)-\max _{y \in \bar{D}} \varphi_{H}(y, T) \\
= & \min _{y \in \bar{D}} \psi_{H}(y)-\max _{y \in \bar{D}} \psi_{H}(y)+\beta T>-\frac{1}{2} \beta T+\beta T=\frac{1}{2} \beta T,
\end{aligned}
$$

that is,

$$
\min _{y \in \bar{D}} \varphi_{H}(y, 0)-\max _{y \in \bar{D}} \varphi_{H}(y, T)>\frac{1}{2} \beta T
$$

for all $H \in \mathcal{H}$.

We set $\varepsilon_{0}<\frac{T}{16}$. Then

$$
\text { if } 0 \leq t \leq 2 \varepsilon_{0} \text {, then } \varphi_{H}(x, t) \geq \varphi_{H}\left(x, 2 \varepsilon_{0}\right) \geq \min _{y \in \bar{D}} \varphi_{H}(y, 0)-2 \varepsilon_{0} \beta
$$


and

$$
\text { if } T-2 \varepsilon_{0} \leq t \leq T \text {, then } \varphi_{H}(x, t) \leq \varphi_{H}\left(x, T-2 \varepsilon_{0}\right) \leq \max _{y \in \bar{D}} \varphi_{H}(y, T)+2 \varepsilon_{0} \beta .
$$

Therefore (3.3) and $\varepsilon_{0}<\frac{T}{16}$ yield

$$
\begin{aligned}
& \min _{x \in \bar{D}, 0 \leq t \leq 2 \varepsilon_{0}} \varphi_{H}(x, t)-\max _{x \in \bar{D}, T-2 \varepsilon_{0} \leq t \leq T} \varphi_{H}(x, t) \\
\geq & \min _{y \in \bar{D}} \varphi_{H}(y, 0)-2 \varepsilon_{0} \beta-\left(\max _{y \in \bar{D}} \varphi_{H}(y, T)+2 \varepsilon_{0} \beta\right) \\
> & \frac{1}{2} \beta T-4 \varepsilon_{0} \beta>\frac{1}{4} \beta T .
\end{aligned}
$$

Setting

$$
\sigma_{1}(H)=\min _{x \in \bar{D}, 0 \leq t \leq 2 \varepsilon_{0}} \varphi_{H}(x, t), \quad \sigma_{2}(H)=\max _{x \in \bar{D}, T-2 \varepsilon_{0} \leq t \leq T} \varphi_{H}(x, t)
$$

we have

$$
\sigma_{1}(H)-\sigma_{2}(H)>\frac{1}{4} \beta T
$$

for all $H \in \mathcal{H}$. Now we define a cut-off function $\chi \in C^{\infty}(\mathbb{R})$ satisfying $0 \leq \chi \leq 1$ and

$$
\chi(t)= \begin{cases}1, & 0<t<T-2 \varepsilon_{0}, \\ 0, & T-\varepsilon_{0}<t<T .\end{cases}
$$

\section{Second Step: Application of Carleman estimate.}

In this step, we apply the Carleman estimate to complete the proof.

Setting

$$
\left\{\begin{array}{l}
v_{k}=u\left(H_{1}, a_{k}\right)-u\left(H_{2}, a_{k}\right), \quad R_{k}=u\left(H_{2}, a_{k}\right), \quad k=1,2, \ldots, n \\
H=H_{2}-H_{1}
\end{array}\right.
$$

we have

$$
\left\{\begin{array}{l}
\partial_{t} v_{k}+\left(H_{1}(x) \cdot \nabla v_{k}\right)+p(x) v_{k}=\left(H(x) \cdot \nabla R_{k}(x, t)\right) \quad \text { in } Q:=D \times(0, T), \\
v_{k}(x, 0)=0 \text { in } D, k=1,2, \ldots, n
\end{array}\right.
$$

Moreover putting

we obtain

$$
w_{k}=\chi \partial_{t} v_{k}, \quad k=1,2, \ldots, n
$$

$$
\left\{\begin{array}{l}
\partial_{t} w_{k}+\left(H_{1}(x) \cdot \nabla w_{k}\right)+p(x) w_{k}=\chi\left(H(x) \cdot \nabla \partial_{t} R_{k}(x, t)\right)+\chi^{\prime}(t) \partial_{t} v_{k} \quad \text { in } Q \\
w_{k}(x, 0)=\left(H(x) \cdot \nabla a_{k}\right) \quad \text { in } D, k=1,2, \ldots, n .
\end{array}\right.
$$

Here and henceforth we write $\chi^{\prime}(t)=\frac{d \chi}{d t}(t)$. For calculating $w_{k}(x, 0)$, we used (3.6) and $R_{k}(x, 0)=u\left(H_{2}, a_{k}\right)(x, 0)=a_{k}(x)$.

By Lemma 2 we have

$$
\left(H_{1}(x) \cdot \nu(x)\right) \leq 0, \quad x \in \partial D \backslash \Gamma .
$$

Therefore, since $w_{k}(\cdot, T)=0$ by (3.5), in terms of Lemma 1 by (3.1), we apply Proposition 1 to $(3.7)$ and we obtain

$$
s \int_{D}\left|w_{k}(x, 0)\right|^{2} e^{2 s \varphi_{H_{1}}(x, 0)} d x+s^{2} \int_{Q}\left|w_{k}\right|^{2} e^{2 s \varphi_{H_{1}}(x, t)} d x d t
$$




$$
\leq C \int_{Q}\left|\chi\left(H(x) \cdot \nabla \partial_{t} R_{k}\right)\right|^{2} e^{2 s \varphi_{H_{1}}} d x d t+C \int_{Q}\left|\chi^{\prime}(t) \partial_{t} v_{k}\right|^{2} e^{2 s \varphi_{H_{1}}} d x d t+C e^{C s} d^{2}
$$

for all $s>s_{0}$. Here and henceforth we set

$$
d=\sum_{k=1}^{n}\left\|w_{k}\right\|_{L^{2}(\Gamma \times(0, T))} \leq \sum_{k=1}^{n}\left\|\partial_{t} u\left(H_{1}, a_{k}\right)-\partial_{t} u\left(H_{2}, a_{k}\right)\right\|_{L^{2}(\Gamma \times(0, T))} .
$$

Since $\chi^{\prime}(t)=0 \forall t \in\left[0, T-2 \varepsilon_{0}\right] \cup\left[T-\varepsilon_{0}, T\right]$, we have

$$
\begin{gathered}
\int_{Q}\left|\chi^{\prime}(t) \partial_{t} v_{k}\right|^{2} e^{2 s \varphi_{H_{1}}} d x d t=\int_{T-2 \varepsilon_{0}}^{T-\varepsilon_{0}} \int_{D}\left|\chi^{\prime}(t)\right|^{2}\left|\partial_{t} u\left(H_{1}, a_{k}\right)-\partial_{t} u\left(H_{2}, a_{k}\right)\right|^{2} e^{2 s \varphi_{H_{1}}} d x d t \\
\leq C e^{2 s \sigma_{2}\left(H_{1}\right)}\left(\left\|\partial_{t} u\left(H_{1}, a_{k}\right)\right\|_{L^{2}(Q)}^{2}+\left\|\partial_{t} u\left(H_{2}, a_{k}\right)\right\|_{L^{2}(Q)}^{2}\right) \leq C e^{2 s \sigma_{2}\left(H_{1}\right)} M_{0}^{2}
\end{gathered}
$$

Substituting the initial value in (3.7) into (3.8) and using the boundedness condition in $\mathcal{H}$ given by (1.3), we see

$$
\begin{aligned}
& s \int_{D}\left|\left(\nabla a_{1}(x), \ldots, \nabla a_{n}(x)\right)^{T} H(x)\right|^{2} e^{2 s \varphi_{H_{1}}(x, 0)} d x \\
\leq & C \int_{0}^{T} \int_{D}|H(x)|^{2} M_{0}^{2} e^{2 s\left(\psi_{H_{1}}(x)-\beta t\right)} d x d t+C e^{2 s \sigma_{2}\left(H_{1}\right)} M_{0}^{2}+C e^{C s} d^{2}
\end{aligned}
$$

for all $s>s_{0}$. By (1.4) we have

$$
\left|\left(\nabla a_{1}(x), \ldots, \nabla a_{n}(x)\right)^{T} H(x)\right| \geq C_{0}|H(x)|, \quad x \in \bar{D}
$$

with some constant $C_{0}>0$. Hence

$$
\begin{aligned}
& s \int_{D}|H(x)|^{2} e^{2 s \varphi_{H_{1}}(x, 0)} d x \\
\leq & C \int_{D}|H(x)|^{2} e^{2 s \varphi_{H_{1}}(x, 0)} d x+C e^{2 s \sigma_{2}\left(H_{1}\right)} M_{0}^{2}+C e^{C s} d^{2}
\end{aligned}
$$

for all $s>s_{0}$. Taking $s_{1}>0$ sufficiently large, we absorb the first term on the right-hand side into the left-hand side, we obtain

$$
s \int_{D}|H(x)|^{2} e^{2 s \varphi_{H_{1}}(x, 0)} d x \leq C e^{2 s \sigma_{2}\left(H_{1}\right)} M_{0}^{2}+C e^{C s} d^{2}
$$

for all $s>s_{1}$. Since $\varphi_{H_{1}}(x, 0) \geq \sigma_{1}\left(H_{1}\right)$ for $x \in \bar{D}$, we have

$$
\|H\|_{L^{2}(D)}^{2} \leq C e^{-2 s\left(\sigma_{1}\left(H_{1}\right)-\sigma_{2}\left(H_{1}\right)\right)} M_{0}^{2}+C e^{C s} d^{2}
$$

for all $s>s_{1}$. The inequality (3.4) implies

$$
\|H\|_{L^{2}(D)}^{2} \leq C e^{-\frac{1}{2} \beta T s} M_{0}^{2}+C e^{C s} d^{2}
$$

for all $s>s_{1}$. Replacing $C$ by $C e^{C s_{1}}$, we see that (3.10) holds for all $s>0$. We then choose $s>0$ so that the right-hand side of (3.10) is smaller. We consider two cases separately.

Case 1: $M_{0}>d$.

Choosing $s>0$ such that

$$
e^{-\frac{1}{2} \beta T s} M_{0}^{2}=e^{C s} d^{2},
$$


that is,

$$
s=\frac{2}{C+\frac{1}{2} \beta T} \log \frac{M_{0}}{d}>0
$$

since $\frac{M_{0}}{d}>1$. Then (3.10) gives

$$
\|H\|_{L^{2}(D)}^{2} \leq 2 C M_{0}^{\frac{4 C}{2 C+\beta T}} d^{\frac{2 \beta T}{2 C+\beta T}}
$$

We set $\theta=\frac{\beta T}{2 C+\beta T} \in(0,1)$.

Case 2: $M_{0} \leq d$.

We readily see that $\|H\|_{L^{2}(D)}^{2} \leq 2 C e^{C s} d^{2}$.

Hence, combining Cases 1 and 2, we reach

$$
\|H\|_{L^{2}(D)} \leq C\left(d^{\theta}+d\right) .
$$

Thus the proof of Theorem 1 is complete.

\section{Proof of Theorem 2}

The proof is similar to Theorem 1 , and simpler because the principal part, i.e. $H(x)$, is known. Setting

$$
y=u\left(p_{1}, a\right)-u\left(p_{2}, a\right), \quad f=p_{1}-p_{2}, \quad R=-u\left(p_{2}, a\right)
$$

we have

$$
\left\{\begin{array}{l}
\partial_{t} y+(H(x) \cdot \nabla y)+p_{1}(x) y=R(x, t) f(x) \quad \text { in } Q:=D \times(0, T), \\
y(x, 0)=0 \text { in } D .
\end{array}\right.
$$

Let $\varepsilon_{0}>0$ be chosen similarly to that of the proof of Theorem 1 in Section 3. We consider $\chi \in C^{\infty}(\mathbb{R}), 0 \leq \chi \leq 1$ defined in (3.5). Putting

$$
z=\chi \partial_{t} y
$$

we obtain

$$
\left\{\begin{array}{l}
\partial_{t} z+(H(x) \cdot \nabla z)+p_{1}(x) z=\chi\left(\partial_{t} R\right)(x, t) f(x)+\chi^{\prime}(t) \partial_{t} y \quad \text { in } Q \\
z(x, 0)=R(x, 0) f(x) \text { in } D .
\end{array}\right.
$$

By Lemma 2, noting that $(H \cdot \nu) \leq 0$ on $\partial D \backslash \Gamma$ implies $\Gamma_{+} \subset \Gamma$, we can apply Proposition 1 to the last system

$$
\begin{gathered}
s \int_{D}|z(x, 0)|^{2} e^{2 s \varphi_{H}(x, 0)} d x+s^{2} \int_{Q}|z|^{2} e^{2 s \varphi_{H}} d x d t \\
\leq C \int_{Q}\left|\chi\left(\partial_{t} R\right) f\right|^{2} e^{2 s \varphi_{H}(x, t)} d x d t+C \int_{Q}\left|\chi^{\prime} \partial_{t} y\right|^{2} e^{2 s \varphi_{H}(x, t)} d x d t+C e^{C s} d_{1}^{2}
\end{gathered}
$$

for all $s>s_{0}$, where we set

$$
d_{1}=\|z\|_{L^{2}(\Gamma \times(0, T)} \leq\left\|\partial_{t} u\left(p_{1}, a\right)-\partial_{t} u\left(p_{2}, a\right)\right\|_{L^{2}(\Gamma \times(0, T))} .
$$


Similarly to (3.9), keeping in mind the definition of $\sigma_{2}(H)$, contained in the proof of Theorem 1 in Section 3, we can estimate

$$
\begin{gathered}
\int_{Q}\left|\chi^{\prime} \partial_{t} y\right|^{2} e^{2 s \varphi_{H}(x, t)} d x d t=\int_{T-2 \varepsilon_{0}}^{T-\varepsilon_{0}} \int_{D}\left|\chi^{\prime} \partial_{t} y\right|^{2} e^{2 s \varphi_{H}(x, t)} d x d t \\
\leq C e^{2 s \sigma_{2}(H)} \int_{T-2 \varepsilon_{0}}^{T-\varepsilon_{0}} \int_{D}\left|\partial_{t} y\right|^{2} d x d t=C e^{2 s \sigma_{2}(H)}\left\|\partial_{t} y\right\|_{L^{2}\left(T-2 \varepsilon_{0}, T-\varepsilon_{0} ; L^{2}(D)\right)}^{2} .
\end{gathered}
$$

Therefore, by assumption (1.7), that is $\left\|\partial_{t} u\left(p_{2}, a\right)\right\|_{L^{2}\left(0, T ; L^{\infty}(D)\right)} \leq M_{0}$, we obtain

$$
\int_{Q}\left|\chi^{\prime} \partial_{t} y\right|^{2} e^{2 s \varphi_{H}(x, t)} d x d t \leq C e^{2 s \sigma_{2}(H)} M_{0}^{2}
$$

Moreover,

$$
\int_{Q}\left|\chi\left(\partial_{t} R\right) f\right|^{2} e^{2 s \varphi_{H}(x, t)} d x d t \leq C M_{0}^{2} \int_{Q}|f(x)|^{2} e^{2 s \varphi_{H}(x, t)} d x d t .
$$

By (4.1), we can calculate

$$
z(x, 0)=\chi(0) \partial_{t} y(x, 0)=R(x, 0) f(x)=-a(x) f(x), \quad x \in D .
$$

Moreover, since

$$
e^{2 s \varphi_{H}(x, t)} \leq e^{2 s \varphi_{H}(x, 0)} \quad \text { for }(x, t) \in Q
$$

the assumption (1.6) yields

$$
s \int_{D}|f(x)|^{2} e^{2 s \varphi_{H}(x, 0)} d x \leq C \int_{D}|f(x)|^{2} e^{2 s \varphi_{H}(x, 0)} d x+C e^{2 s \sigma_{2}(H)} M_{0}^{2}+C e^{C s} d_{1}^{2}
$$

for all $s>s_{0}$. Therefore similarly to (3.10), we can obtain

$$
\|f\|_{L^{2}(D)}^{2} \leq C e^{-\frac{1}{2} \beta T s} M_{0}^{2}+C e^{C s} d_{1}^{2}
$$

for all $s>s_{0}$. We can follow the proof of Theorem 1 , and the proof of Theorem 2 is complete.

\section{Closing Remarks}

In this section, we discuss the determination of both coefficients and solution to the transport equation also with other formulations of observation data. For simplicity, we consider linearized problems of inverse problems of determining a zeroth order coefficient $p$ in (1.1). We can similarly study non-linearized inverse problems of determining a vector-valued function $H(x)$, but we here omit details.

We set $y=u\left(p_{1}, a\right)-u\left(p_{2}, a\right)$. Using the notations of the previous sections, we consider

$$
\left\{\begin{array}{l}
\partial_{t} y(x, t)+(H(x) \cdot \nabla y)+p_{1}(x) y(x, t)=R(x, t) f(x), \quad x \in D, 0<t<T, \\
y(x, 0)=0, \quad x \in D .
\end{array}\right.
$$

Henceforth we assume that $H \in \mathcal{H}, p_{1} \in L^{\infty}(D), R \in H^{1}\left(0, T ; L^{\infty}(D)\right)$ are fixed and $f \in L^{2}(D)$, and $R(x, 0) \neq 0$ on $\bar{D}$. Then, in terms of Lemma 2 , by the same argument in 
First Step of the proof of Theorem 1, we can choose a domain $D$ such that

$$
\left\{\begin{array}{l}
D \text { depends on } H, T, \Omega, \quad \partial D=\overline{\Gamma_{+} \cup \Gamma_{-}}, \\
\text {where } \Gamma_{-}:=\{x \in \partial D ; H(x) \cdot \nu(x) \leq 0\}, \quad \Gamma_{+}:=\{x \in \partial D ; H(x) \cdot \nu(x) \geq 0\} \subset \Gamma, \\
\Gamma_{+} \cap \Gamma_{-} \text {has no interior points, } \quad x_{0} \in \partial D \cap \partial \Omega \subset \Gamma .
\end{array}\right.
$$

We note that the domain $D$ can depend on $H$ and fixing $H$, we can consider that $D$ is fixed for our discussions in this section.

\section{§5.1. Determination of solution $y(x, t)$.}

In Theorems 1 and 2, we are restricted to the determination of coefficients, and we do not consider the determination of solution $u$ itself to (1.1). For the linearized inverse problem, the same argument as the proof of Theorem 2 yields conditional Hölder stability in determing $f$ in $D$ and $y$ in $D \times(0, \varepsilon)$ where $\varepsilon>0$ is sufficiently small.

\section{Proposition 2.}

There exist constants $C>0, \theta_{1} \in(0,1)$ and small $\varepsilon>0$ such that

$$
\|f\|_{L^{2}(D)}+\|y\|_{H^{1}\left(0, \varepsilon ; L^{2}(D)\right)} \leq C\left(\left\|\partial_{t} y\right\|_{L^{2}(\Gamma \times(0, T))}^{\theta_{1}}+\left\|\partial_{t} y\right\|_{L^{2}(\Gamma \times(0, T))}\right)
$$

provided that $\left\|\partial_{t} y\right\|_{L^{2}\left(0, T ; L^{\infty}(D)\right)} \leq M_{0}$ with arbitrarily fixed constant $M_{0}>0$. The constants $C>0$ and $\theta_{1} \in(0,1), \varepsilon>0$ depend on $\Omega, T, \Gamma, H$.

The interval length $\varepsilon$ for estimating $y$ is small, which means that the estimate is only near $t=0$. As Example in Section 1 shows, we cannot prove $\varepsilon=T$.

Proof of Proposition 2. The estimation of $\|f\|_{L^{2}(D)}$ is the same as Theorem 2. We recall that $Q=D \times(0, T)$. Now we estimate $y$. By the same manner as in (4.2) and (4.4), we obtain

$$
\begin{aligned}
& s^{2} \int_{Q}\left|\chi \partial_{t} y\right|^{2} e^{2 s \varphi_{H}(x, t)} d x d t \\
\leq & C \int_{Q}\left|\chi\left(\partial_{t} R\right) f\right|^{2} e^{2 s \varphi_{H}(x, t)} d x d t+C e^{2 s \sigma_{2}(H)} M_{0}^{2}+C e^{C s} d_{1}^{2} \\
\leq & C e^{2 C s}\|f\|_{L^{2}(D)}^{2}+C e^{2 s \sigma_{2}(H)} M_{0}^{2}+C e^{C s} d_{1}^{2} .
\end{aligned}
$$

Here we used $\partial_{t} R \in L^{2}\left(0, T ; L^{\infty}(D)\right)$ and we set

$$
d_{1}=\left\|\partial_{t} y\right\|_{L^{2}(\Gamma \times(0, T))} .
$$

Since $\varphi_{H}(x, t) \geq \sigma_{1}(H)$ for $x \in D$ and $0 \leq t \leq 2 \varepsilon_{0}=: \varepsilon$ and $\chi(t)=1$ for $0 \leq t \leq 2 \varepsilon_{0}$, we have

$$
s^{2} e^{2 s \sigma_{1}(H)}\left\|\partial_{t} y\right\|_{L^{2}\left(0, \varepsilon ; L^{2}(D)\right)}^{2} \leq C e^{2 C s}\|f\|_{L^{2}(D)}^{2}+C e^{2 s \sigma_{2}(H)} M_{0}^{2}+C e^{C s} d_{1}^{2} .
$$

Since we have already proved $\|f\|_{L^{2}(D)} \leq C\left(d_{1}+d_{1}^{\theta}\right)$, we reach

$$
\left\|\partial_{t} y\right\|_{L^{2}\left(0, \varepsilon ; L^{2}(D)\right)}^{2} \leq C e^{2 C s}\left(d_{1}^{2}+d_{1}^{2 \theta}\right)+C e^{-2 s\left(\sigma_{1}(H)-\sigma_{2}(H)\right)} M_{0}^{2}
$$

for all large $s>0$. By (3.4), we can argue in the same was as for (3.10), and we complete the estimate of $\left\|\partial_{t} y\right\|_{L^{2}\left(0, \varepsilon ; L^{2}(D)\right)}$ with some $\theta_{1} \in(0, \theta)$. Since $y(\cdot, 0)=0$ in $D$, we easily prove 
$\|y\|_{H^{1}\left(0, \varepsilon ; L^{2}(D)\right)} \leq C\left\|\partial_{t} y\right\|_{L^{2}\left(0, \varepsilon ; L^{2}(D)\right)}$. Thus the proof of Proposition 2 is complete.

\section{§5.2. Other formulations of the inverse problem}

So far we formulate the inverse problem with data of $y$ on $D \times\{0\}$ and $\Gamma_{+} \times(0, T)$. As possible data sets, we can introduce:

- Case I: $D \times\{T\}$ and $\Gamma_{-} \times(0, T)$

- Case II: $D \times\{0\}$ and $\Gamma_{-} \times(0, T)$

- Case III: $D \times\{T\}$ and $\Gamma_{+} \times(0, T)$

- Case IV: $D \times\{0, T\}$ and $\Gamma_{+} \times(0, T)$

- Case V: $D \times\{0, T\}$ and $\Gamma_{-} \times(0, T)$

- Case VI: $D \times\{0\}$ and $\left(\Gamma_{-} \cup \Gamma_{+}\right) \times(0, T)$

- Case VII: $D \times\{T\}$ and $\left(\Gamma_{-} \cup \Gamma_{+}\right) \times(0, T)$

We note that we exclude the two cases of data on $\left(\Gamma_{-} \cup \Gamma_{+}\right) \times(0, T)$ and $D \times\{0, T\}$, because we can expect that such two data sets are both too poor and that we cannot prove any uniqueness for the corresponding invers problems.

By the change of variables $t \longrightarrow T-t$, setting $v(x, t)=u(x, T-t)$ for $x \in D$ and $0<t<T$, we have $\partial_{t} v(x, t)=-\partial_{t} u(x, T-t)$, so that (5.1) can be written in terms of $v$ and $-H(x)$. Therefore we can exchange $\Gamma_{+}$by $\Gamma_{-}, \Gamma_{-}$by $\Gamma_{+}, u(x, 0)$ by $v(x, T)$. Hence Case I is equivalent to our currently considered case of data on $D \times\{0\}$ and $\Gamma_{+} \times(0, T)$, Case II to Case III, Case IV to Case V, Case VI to Case VII. Thus it is sufficient to discuss the three Cases II, IV. VI.

\section{Case II.}

In Case II, we do not know positive results for the inverse problem by our method. In fact, we cannot prove an adequate Carleman estimate which corresponds to Propostion 1, and for the proof of Carleman estimate in Case II, the signs of $B_{H}(x, 0)$ and $B_{H}(x)(H(x) \cdot \nu)$ on $\Gamma_{-} \times(0, T)$ are contradictive.

Data on $\Gamma_{+} \times(0, T)$ may be meaningful for determining $f$. On the other hand, data on $\Gamma_{-} \times(0, T)$ are not meaningful for $f$, but with given $f$, data on $\Gamma_{-} \times(0, T)$ and $y(\cdot, 0)$ perfectly determine $y$ on $D \times(0, T)$ by a usual energy estimate (Lemma 3 below).

\section{Case IV.}

The result is different and we can prove an unconditional Lipschitz stability.

\section{Proposition 3.}

There exists a constant $C>0$ such that

$$
\|f\|_{L^{2}(D)}+\|y\|_{H^{1}\left(0, T ; L^{2}(D)\right)} \leq C\left(\left\|\partial_{t} y\right\|_{L^{2}(\Gamma \times(0, T))}+\|y(\cdot, T)\|_{H^{1}(D)}\right) .
$$

Here the constant $C>0$ is independent of any bounds of $y$ and dependent on $\Omega, T, \Gamma, H$, which means that the stability is unconditional.

Proof.

Since we are given $y(\cdot, T)$, we need not the cut-off function $\chi$ satisfying (3.5). Setting 
$y_{1}=\partial_{t} y$, we differentiate the first equation in (5.1) to have

$$
\left\{\begin{array}{l}
\partial_{t} y_{1}(x, t)+\left(H(x) \cdot \nabla y_{1}\right)+p_{1}(x) y_{1}(x, t)=\left(\partial_{t} R(x, t)\right) f(x), \quad x \in D, 0<t<T, \\
y_{1}(x, 0)=R(x, 0) f(x), \quad x \in D
\end{array}\right.
$$

and

$$
y_{1}(x, T)=-(H(x) \cdot \nabla y(x, T))-p_{1}(x) y(x, T)+R(x, T) f(x), \quad x \in D .
$$

Applying Proposition 1, we obtain

$$
\begin{aligned}
& s \int_{D}|R(x, 0)|^{2}|f(x)|^{2} e^{2 s \varphi_{H}(x, 0)} d x+s^{2} \int_{Q}\left|\partial_{t} y\right|^{2} e^{2 s \varphi_{H}(x, t)} d x d t \\
\leq & C \int_{Q}\left|\partial_{t} R\right|^{2}|f|^{2} e^{2 s \varphi_{H}(x, t)} d x d t+C s \int_{D}\left|(H(x) \cdot \nabla y(x, T))+p_{1}(x) y(x, T)\right|^{2} e^{2 s \varphi_{H}(x, T)} d x \\
+ & C s \int_{D}|R(x, T)|^{2}|f|^{2} e^{2 s \varphi_{H}(x, T)} d x+C e^{C s} d_{1}^{2}
\end{aligned}
$$

for all large $s>0$. Here we recall that $d_{1}$ is defined by (5.4).

By $\partial_{t} R \in L^{2}\left(0, T ; L^{\infty}(D)\right)$ and $R(x, 0) \neq 0$ for $x \in \bar{D}$, since $\varphi_{H}(x, t) \leq \varphi_{H}(x, 0)$ for $(x, t) \in Q$ and $\sigma_{2}(H) \geq \max _{x \in \bar{D}} \varphi_{H}(x, T)$, we obtain

$$
\begin{aligned}
& s \int_{D}|f(x)|^{2} e^{2 s \varphi_{H}(x, 0)} d x+s^{2} e^{-2 s C_{1}}\left\|\partial_{t} y\right\|_{L^{2}\left(0, T ; L^{2}(D)\right)}^{2} \\
\leq & C \int_{D}|f(x)|^{2} e^{2 s \varphi_{H}(x, 0)} d x+C s e^{2 s C_{1}}\|y(\cdot, T)\|_{H^{1}(D)}^{2}+C s e^{2 s \sigma_{2}(H)}\|f\|_{L^{2}(D)}^{2}+C e^{C s} d_{1}^{2} .
\end{aligned}
$$

Here we set $C_{1}=\left\|\varphi_{H}\right\|_{L^{\infty}(Q)}$. Choosing $s>0$ large, we can absorb the first term on the right-hand side into the left-hand side. Using

$$
\varphi_{H}(x, 0) \geq \min _{x \in \bar{D}} \varphi_{H}(x, 0) \geq \sigma_{1}(H),
$$

and dividing by $e^{2 s \sigma_{1}(H)}$, we reach

$$
\begin{aligned}
& \|f\|_{L^{2}(D)}^{2}+s^{2} e^{-2 s\left(C_{1}+\sigma_{1}(H)\right)}\left\|\partial_{t} y\right\|_{L^{2}\left(0, T ; L^{2}(D)\right)}^{2} \\
\leq & C s e^{2 s C_{1}}\|y(\cdot, T)\|_{H^{1}(D)}^{2}+C s e^{-2 s\left(\sigma_{1}(H)-\sigma_{2}(H)\right)}\|f\|_{L^{2}(D)}^{2}+C e^{C s} d_{1}^{2}
\end{aligned}
$$

for all large $s>0$. In terms of (3.4), again choosing $s>0$ large, we can absorb the second term on the right-hand side into the left-hand side, and we complete the proof of Proposition 3.

\section{Case VI.}

Also in this case, we can prove an unconditional Lipschitz stability estimate but the norm of $y$ is not the same.

\section{Proposition 4.}

There exists a constant $C>0$ such that

$$
\|f\|_{L^{2}(D)}+\|y\|_{W^{1, \infty}\left(0, T ; L^{2}(D)\right)} \leq C\left\|\partial_{t} y\right\|_{L^{2}(\partial D \times(0, T))}
$$

with some constant $C>0$ which is independent of any bounds of $\left\|\partial_{t} y\right\|_{L^{2}\left(0, T ; L^{\infty}(D)\right)}$.

Proof. 
With the same notation $z=\chi \partial_{t} y$, in the proof of Theorem 2, we can obtain (4.2) and (4.3), where $d_{1}$ is defined by

$$
d_{1}=\left\|\chi \partial_{t} y\right\|_{L^{2}\left(\Gamma_{+} \times(0, T)\right)}
$$

Therefore

$$
\begin{aligned}
& s \int_{D}\left|\partial_{t} y(x, 0)\right|^{2} e^{2 s \varphi_{H}(x, 0)} d x \leq C \int_{Q}|f(x)|^{2} e^{2 s \varphi_{H}(x, t)} d x d t \\
+ & C e^{2 s \sigma_{2}(H)}\left\|\partial_{t} y\right\|_{L^{2}\left(T-2 \varepsilon_{0}, T-\varepsilon_{0} ; L^{2}(D)\right)}^{2}+C e^{C s} d_{1}^{2}
\end{aligned}
$$

for all large $s>0$. Since $\partial_{t} y(x, 0)=R(x, 0) f(x)$ and $\varphi_{H}(x, t) \leq \varphi_{H}(x, 0)$ for $(x, t) \in Q$, we have

$$
\begin{aligned}
& s \int_{D}|f(x)|^{2} e^{2 s \varphi_{H}(x, 0)} d x \leq C \int_{D}|f(x)|^{2} e^{2 s \varphi_{H}(x, 0)} d x d t \\
+ & C e^{2 s \sigma_{2}(H)}\left\|\partial_{t} y\right\|_{L^{2}\left(T-2 \varepsilon_{0}, T-\varepsilon_{0} ; L^{2}(D)\right)}^{2}+C e^{C s} d_{1}^{2}
\end{aligned}
$$

Choosing $s>0$ large, we can absorb the first term on the right-hand side into the left-hand side, we obtain

$$
\int_{D}|f(x)|^{2} e^{2 s \varphi_{H}(x, 0)} d x \leq C e^{2 s \sigma_{2}(H)}\left\|\partial_{t} y\right\|_{L^{2}\left(0, T ; L^{2}(D)\right)}^{2}+C e^{C s} d_{1}^{2}
$$

The following lemma is usual energy estimation and estimates $\left\|\partial_{t} y\right\|_{L^{2}\left(0, T ; L^{2}(D)\right)}^{2}$.

\section{Lemma 3.}

There exists a constant $C>0$ depending on $R$, such that

$$
\left\|\partial_{t} y(\cdot, t)\right\|_{L^{2}(D)} \leq C\left(\|f\|_{L^{2}(D)}+\left\|\partial_{t} y\right\|_{L^{2}\left(\Gamma_{-} \times(0, T)\right)}\right), \quad 0 \leq t \leq T
$$

\section{Proof of Lemma 3.}

Setting $y_{1}=\partial_{t} y$, we have (5.5). Multiplying the first equation in (5.5) with $2 y_{1}$ and integrating in $D$, we have

$$
\begin{aligned}
& \partial_{t} \int_{D}\left|y_{1}(x, t)\right|^{2} d x+2 \int_{D} \sum_{j=1}^{n} h_{j}(x)\left(\partial_{j} y_{1}(x, t)\right) y_{1}(x, t) d x+2 \int_{D} p_{1}(x)\left|y_{1}(x, t)\right|^{2} d x \\
= & 2 \int_{D}\left(\partial_{t} R\right)(x, t) f(x) y_{1}(x, t) d x .
\end{aligned}
$$

Here the integration by parts provides

$$
\begin{aligned}
& 2 \int_{D} \sum_{j=1}^{n} h_{j}(x)\left(\partial_{j} y_{1}(x, t)\right) y_{1}(x, t) d x=\int_{D} \sum_{j=1}^{n} h_{j}(x) \partial_{j}\left(\left|y_{1}(x, t)\right|^{2}\right) d x \\
= & \int_{\partial D}(H \cdot \nu)\left|y_{1}\right|^{2} d S-\int_{D}(\operatorname{div} H)\left|y_{1}(x, t)\right|^{2} d x \\
\geq & \int_{\Gamma_{-}}(H \cdot \nu)\left|y_{1}\right|^{2} d S-\int_{D}(\operatorname{div} H)\left|y_{1}(x, t)\right|^{2} d x
\end{aligned}
$$


Setting $E(t)=\int_{D}\left|y_{1}(x, t)\right|^{2} d x$, the Cauchy-Schwarz inequality yields

$$
\begin{aligned}
& \frac{d E(t)}{d t}-C \int_{\Gamma_{-}}\left|y_{1}\right|^{2} d S \leq C E(t)+\int_{D}\left|\left(\partial_{t} R\right) f\right|^{2} d x+\int_{D}\left|y_{1}\right|^{2} d x \\
\leq & C E(t)+C\|f\|_{L^{2}(D)}^{2} .
\end{aligned}
$$

Therefore by integration, we have

$$
E(t) \leq E(0)+C \int_{0}^{t} E(\eta) d \eta+C\left(\left\|y_{1}\right\|_{L^{2}\left(\Gamma_{-} \times(0, T)\right)}^{2}+\|f\|_{L^{2}(D)}^{2}\right), \quad 0 \leq t \leq T .
$$

Since $E(0)=\int_{D}|R(x, 0) f(x)|^{2} d x \leq C\|f\|_{L^{2}(D)}^{2}$, the Gronwall inequality completes the proof of Lemma 3.

Now we return to the proof of Proposition 4. Applying Lemma 3 in the first term on the right-hand side of (5.6), we have

$$
\begin{aligned}
& \int_{D}|f(x)|^{2} e^{2 s \varphi_{H}(x, 0)} d x \leq C e^{2 s \sigma_{2}(H)}\|f\|_{L^{2}(D)}^{2} \\
+ & C e^{2 s \sigma_{2}(H)}\left\|\partial_{t} y\right\|_{L^{2}\left(\Gamma_{-} \times(0, T)\right)}^{2}+C e^{C s}\left\|\partial_{t} y\right\|_{L^{2}\left(\Gamma_{+} \times(0, T)\right)}^{2}
\end{aligned}
$$

for all large $s>0$. Noting (3.4) and using $\varphi_{H}(x, 0) \geq \sigma_{1}(H)$ for $x \in D$, we divide both sides with $e^{2 s \sigma_{1}(H)}$, we obtain

$$
\|f\|_{L^{2}(D)}^{2} \leq C e^{-\frac{1}{2} \beta T s}\|f\|_{L^{2}(D)}^{2}+C e^{C s}\left\|\partial_{t} y\right\|_{L^{2}\left(\left(\Gamma_{+} \cup \Gamma_{-}\right) \times(0, T)\right)}^{2}
$$

for all large $s>0$. Again choosing $s>0$ sufficiently large, we can absorb the first term on the right-hand side into the left-hand side, so that

$$
\|f\|_{L^{2}(D)}^{2} \leq C e^{C s}\left\|\partial_{t} y\right\|_{L^{2}(\partial D \times(0, T))}^{2} .
$$

With Lemma 3, we see also

$$
\left\|\partial_{t} y\right\|_{L^{\infty}\left(0, T ; L^{2}(D)\right)} \leq C\left\|\partial_{t} y\right\|_{L^{2}(\partial D \times(0, T))} .
$$

Thus the proof of Proposition 4 is completed.

Acknowledgement. This work was partially supported by Grant-in-Aid for Scientific Research (S) $15 \mathrm{H} 05740$ of Japan Society for the Promotion of Science, and by NSFC (No. 11771270, 91730303) and the "RUDN University Program 5-100". The first and second authors were visitors at The University of Tokyo in February 2018, supported by the above grant of the Japan Society for the Promotion of Science. Most of the paper was completed during the stay of the fourth author as GNAMPA visitor at the IN $\delta$ AM Unit of the Università degli Studi di Napoli Federico II in January 2019 and he is grateful for the supports. The fourth author was a Visiting Scholar at Rome in April 2018 supported by the University of Rome "Tor Vergata". This work was supported also by the Istituto Nazionale di Alta Matematica (INdAM), through the GNAMPA Research Project 2017 "Comportamento asintotico e controllo di equazioni di evoluzione non lineari". Moreover, this research was performed within the framework of the French-German-Italian Laboratoire International Associé (LIA), named COPDESC, on Applied Analysis, issued by CNRS, MPI and INdAM. 
This work was also supported by the research project of the Università di Napoli Federico II: "Spectral and Geometrical Inequalities".

\section{REFERENCES}

[1] L. Beilina and M. V. Klibanov, Approximate Global Convergence and Adaptivity for Coefficient Inverse Problems, Springer-Verlag, Berlin, 2012.

[2] S. P. Belinskij, On one of the inverse problems for linear symmetrical t-hyperbolic systems with $n+1$ independent variables, Differential Equations 12 (1976) 15-23.

[3] M. Bellassoued and M. Yamamoto, Carleman Estimates and Applications to Inverse Problems for Hyperbolic Systems, Springer-Japan, Tokyo, 2017.

[4] A.L. Bukhgeim and M.V. Klibanov, Global uniqueness of a class of multidimensional inverse problems, Sov. Math. Dokl. 24, 244-247.

[5] P. Cannarsa, G. Floridia and M. Yamamoto, Observability inequalities for transport equations through Carleman estimates, preprint, https://arxiv.org/abs/1807.05005.

[6] P. Gaitan and H. Ouzzane, Inverse problem for a free transport equation using Carleman estimates, Appl. Anal. 93 (2014) 1073-1086.

[7] F. Gölgeleyen and M. Yamamoto, Stability for some inverse problems for transport equations, SIAM J. Math. Anal. 48 (2016) 2319-2344.

[8] L. Hörmander, Linear Partial Differential Operators, Springer-Verlag, Berlin, 1963.

[9] O.Y. Imanuvilov and M. Yamamoto, Lipschitz stability in inverse parabolic problems by the Carleman estimate, Inverse Problems 14 (1998) 1229-1245.

[10] O.Y. Imanuvilov an M. Yamamoto, Global Lipschitz stability in an inverse hyperbolic problem by interior observations, Inverse Problems 17 (2001) 717-728.

[11] O.Y. Imanuvilov and M. Yamamoto, Determination of a coefficient in an acoustic equation with single measurement, Inverse Problems 19 (2003) 157-171.

[12] O.Y. Imanuvilov and M. Yamamoto, Carleman estimates for the Lamé system with stress boundary condition, Publ. Res. Inst. Math. Sci. 43 (2007) 1023-1093.

[13] V.Isakov, Inverse Problems for Partial Differential Equations, Springer-Verlag, Berlin, 2006.

[14] M.V. Klibanov, Inverse problems and Carleman estimates, Inverse Problems 8 (1992) 575-596.

[15] M.V. Klibanov, Carleman estimates for global uniqueness, stability and numerical methods for coefficient inverse problems, J. Inverse Ill-Posed Probl. 21 (2013) 477-560.

[16] M.V. Klibanov and S.E. Pamyatnykh, Lipschitz stability of a non-standard problem for the nonstationary transport equation via a Carleman estimate, Inverse Problems 22 (2006) 881-890.

[17] M.V. Klibanov and S.E. Pamyatnykh, Global uniqueness for a coefficient inverse problem for the nonstationary transport equation via Carleman estimate, J. Math. Anal. Appl. 343 (2008) 352-365. 
[18] M.V. Klibanov and A. Timonov, Carleman Estimates for Coefficient Inverse Problems and Numerical Applications, VSP, Utrecht, 2004.

[19] M.V. Klibanov and M. Yamamoto, Lipschitz stability of an inverse problem for an accoustic equation, Appl. Anal. 85 (2006) 515-538.

[20] M. Machida and M. Yamamoto, Global Lipschitz stability in determining coefficients of the radiative transport equation, Inverse Problems 30 (2014) 035010.

[21] V. G. Romanov, Inverse Problems of Mathematical Physics, VNU Science Press, Utrecht, the Netherlands, 1987.

[22] M. Yamamoto, Carleman estimates for parabolic equations and applications. Inverse Problems 25 (2009) 123013. 